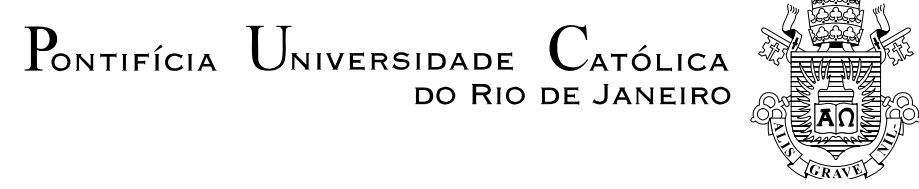

Tarso Mori Madeira

Coordenação entre pequenos fundos de investimento em um mercado relativamente ilíquido: efeitos sobre volume e preços

Dissertação de Mestrado

Dissertação apresentada ao Programa de Pós-Graduação em Economia do Departamento de Economia da PUC-Rio como parte dos requisitos parciais para obtenção do título de Mestre em Economia.

Orientador: Vinicius do Nascimento Carrasco

Rio de Janeiro

Março de 2009 


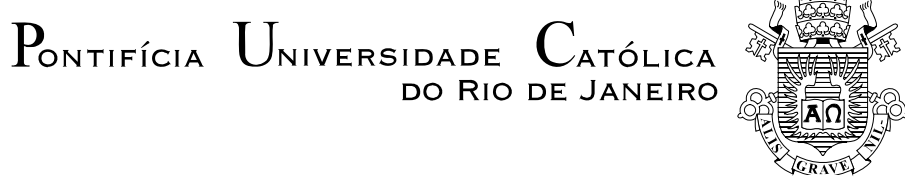

Tarso Mori Madeira

\title{
Coordenação entre pequenos fundos de investimento em um mercado relativamente ilíquido: efeitos sobre volume e preços
}

Dissertação apresentada como requisito parcial para obtenção do grau de Mestre pelo Programa de PósGraduação em Economia do Departamento de Economia do Centro de Ciências Sociais da PUC-Rio. Aprovada pela Comissão Examinadora abaixo assinada.

\author{
Prof. Vinicius do Nascimento Carrasco \\ Orientador \\ Departamento de Economia - PUC-Rio
}

Prof. Walter Novaes Filho

Departamento de Economia - PUC-Rio

Prof. Luis Henrique Bertolino Braido Escola de Pós-Graduação em Economia - FGV/RJ

Prof. Nizar Messari

Coordenador(a) Setorial do Centro de Ciências Sociais - PUC-Rio

Rio de Janeiro, 25 de março de 2009 
Todos os direitos reservados. É proibida a reprodução total ou parcial do trabalho sem autorização do autor, do orientador e da universidade.

\section{Tarso Mori Madeira}

Graduou-se em Ciências Econômicas na Universidade Federal do Rio Grande do Sul em 2006. Atual aluno do programa de Doutorado em Economia da University of California, Berkeley.

\section{Ficha Catalográfica}

Coordenação entre pequenos fundos de investimento em um mercado relativamente ilíquido: efeitos sobre volume e preços / Tarso Mori Madeira ; orientador: Vinicius do Nascimento Carrasco. - 2009.

54 f. ; $30 \mathrm{~cm}$

1.Dissertação (Mestrado) - Pontifícia Universidade Católica do Rio de Janeiro, Departamento de Economia, 2009.

Inclui bibliografia

1. Economia - Teses. 2. Volume. 3. Volatilidade de preço. 4. Fundos de investimento. 5. Carteira de investimento. 6. Complementaridade. 7. Coordenação. 8. Concurso de rentabilidade. I. Carrasco, Vinicius do Nascimento. II. Pontifícia Universidade Católica do Rio de Janeiro. Departamento de Economia. III. Título. 


\section{Agradecimentos}

Ao meu orientador Vinicius do Nascimento Carrasco pelos ensinamentos, pela dedicação e paciência.

Aos Professores Walter Novaes Filho e Luis Henrique Bertolino Braido pelas críticas e sugestões.

À CAPES pelos auxílios concedidos.

À Ana Maria Mori Madeira pelo suporte.

A todos que de uma forma ou de outra me estimularam ou me ajudaram. 


\section{Resumo}

Madeira, Tarso Mori.; Carrasco, Vinicius do Nascimento. Coordenação entre pequenos fundos de investimento em um mercado relativamente ilíquido: efeitos sobre volume e preços. Rio de Janeiro, 2009. 54p. Dissertação de Mestrado - Departamento de Economia, Pontifícia Universidade Católica do Rio de Janeiro.

Este trabalho é composto por dois artigos. No primeiro deles, modela-se a atuação de fundos de investimento pequenos em mercados relativamente ilíquidos com o objetivo de identificar os efeitos de suas transações sobre o volume transacionado das carteiras de ativos, bem como sobre seus preços. Insere-se um incentivo à coordenação entre os fundos de investimento, o que faz com que a distribuição das carteiras compostas pelos fundos seja concentrada ao redor da carteira média de mercado. Como se lida com um mercado ilíquido, tem-se que as carteiras próximas à média sofrerão um aumento maior de preço. Além disso, mostra-se que a quantidade total - soma das quantidades investidas em todas as carteiras por todos os fundos - cresce quando o motivo de coordenação está presente. Esse crescimento contribui para o aumento médio da variação de preços dentre todas as carteiras. O segundo artigo se dedica à inclusão no modelo básico de uma nova fonte de informação quanto à rentabilidade das carteiras dos fundos: um concurso de rentabilidade. Mostra-se que os resultados alcançados são exatamente opostos aos do aumento da coordenação.

\section{Palavras-chave}

Volume; volatilidade de preço; fundos de investimento; carteira de investimento; complementaridade; coordenação; concurso de rentabilidade; ranking. 


\section{Abstract}

Madeira, Tarso Mori.; Carrasco, Vinicius do Nascimento (advisor). Coordination among small investment funds in a relatively illiquid market: effects on volume and prices. Rio de Janeiro, 2009. 54p. MSc Dissertation - Departamento de Economia, Pontifícia Universidade Católica do Rio de Janeiro.

The present work is formed by two articles. In the first, I model the interaction of small investment funds in a relatively illiquid market with the aim of identifying the effects of their transactions on volume and price movements of assets. The behavior of the investment funds exhibits a coordination-motive, which ultimately diminishes the differences in the composition of the various portfolios. In an illiquid market, the rise of the price of these portfolios is higher. Besides, I show that the total volume bought by all funds rises when the coordination-motive is present. That contributes to raise the variation of prices among all portfolios. The second article is dedicated to the inclusion on the basic model of a new source of information concerning the profitability of the funds: a profitability contest. The results are opposite to the case in which the coordination-motive is present.

\section{Keywords}

Volume; price volatility; investment funds; portfolio; complementarity, coordination; profitability contest; ranking. 


\section{Sumário}

1 Introdução

2 Coordenação entre pequenos fundos de investimento em um mercado relativamente ilíquido: efeitos sobre volume e preços

2.1. Motivação 11

$\begin{array}{ll}\text { 2.2. Modelo } & 14\end{array}$

$\begin{array}{ll}\text { 2.2.1. Fundos de investimento } & 14\end{array}$

$\begin{array}{ll}\text { 2.2.2. Provedores de liquidez } & 15\end{array}$

$\begin{array}{ll}\text { 2.2.3. Preço das carteiras } & 16\end{array}$

$\begin{array}{ll}\text { 2.3. Estrutura informacional } & 16\end{array}$

$\begin{array}{lr}\text { 2.4. Timing } & 18\end{array}$

2.5. Variação do preços das carteiras em equilíbrio 18

2.6. Estratégia dos fundos de investimento 21

2.6.1. Quantidade de recursos administrada 22

2.6.2. Carteira escolhida 23

2.6.2.1. Carteira que maximiza a rentabilidade 24

$\begin{array}{ll}\text { 2.7. Resultados } & 26\end{array}$

3 Concurso de Rentabilidade 31

3.1. Motivação 31

3.2. Modificações no modelo 32

3.3. Equilíbrio do jogo de maximização da publicidade 33

3.4. Equilíbrio do jogo de maximização da rentabilidade esperada pelo público $\quad 35$

3.5. Resultados 36

4 Conclusões $\quad 39$

5 Referências bibliográficas 41 
6 Apêndice

6.1. Introdução de um fundo de investimento grande

6.2. Demonstrações 